EPJ Web of Conferences 33, 05010 (2012)

DOI: $10.1051 /$ epjconf $/ 20123305010$

C) Owned by the authors, published by EDP Sciences, 2012

\title{
Technical and economic analysis of a Smart Public Lighting model
}

\author{
F. Bucci ${ }^{1}$, M. Annunziato ${ }^{2}$, and F. Moretti ${ }^{3}$ \\ ${ }^{1}$ Citelum SA, Via C. Monteverdi 11, 20131 Milan, Italy, fbucci@citelum.com \\ ${ }^{2}$ Energy New technologies and sustainable Economic development Agency (ENEA), \\ Casaccia R.C., Via Anguillarese 301, 00123 Rome, Italy, mauro.annunziato@enea.it \\ ${ }^{3}$ University of Rome "Roma Tre", Via della Vasca Navale, 79/81, 00146 Rome, Italy, \\ fabiomor@gmail.com
}

\begin{abstract}
The study focuses on public lighting (PL) and is based on data collected through the Lumière project (www.progettolumiere.enea.it) (ENEA). We identified three intervention strategies that have been piloted in the town of Castelnuovo: 1) Line control 2) Remote point-to-point control 3) Smart (or Adaptive) Lighting. The three solutions were compared according to their net present value (NPV) and payback time (PBT) and considering the energy saving and the security level. Overall, the third solution was found to be the best. The study was extended, given certain assumptions, to the municipalities in the Lumière Network and to Italy, and some very interesting results emerged. In conclusion, an adaptive control system would substantially increase Italy's energy efficiency and would also significantly reduce municipalities' expenditure. It would also represent a significant development in the field of PL as well as in transport, building management, security and the quality of services offered to citizens and tourists in general.
\end{abstract}

\section{Introduction}

It is very important to focus attention on public lighting (PL) in order to reduce electricity consumption, for three reasons: 1) the high total volume of energy (at $6.1 \mathrm{TWh}, \mathrm{PL}$ represents $22 \%$ of Italian energy demand for lighting [1]), 2) PL is the third largest expense for Italian municipalities, 3) the potential energy savings (which range around 30-40\%). New technologies that promise higher energy efficiency have recently emerged (such as LEDs and advanced control systems) but their introduction must be supported by in-depth technical and economic analysis to make the promise of increased performance credible.

The aim of this work is to assess the efficacy and competitiveness of technologically innovative solutions that are placed within the broader context of the 'Smart City' [2], offering the possibility to transform the PL network into a digital and electrical 
infrastructure with widespread diffusion in the city and to integrate so-called 'Smart' Services into the PL network [3].

The study was based on data collected through the Lumière project (ENEA) (www.progettolumiere.enea.it) which aims at helping municipalities improve the efficiency of PL systems [4] by creating technical and economic evaluation tools and methodologies to analyze the PL system of small and medium towns.

Analyzing existing technologies [5] we identified three intervention strategies that have been piloted. These are:

1. Line control (LC)

2. Remote point-to-point control [6] (P2P)

3. Smart (or Adaptive) Lighting (SL)

The comparison showed that the more attractive strategy, both from the point of view of energy saving and of competitiveness, is the adaptive control of illumination. This consists of supplying lighting in a way proportional to the demand (flows of vehicles and people). This approach, which is still being piloted, can produce an average energy saving of more than $40 \%$, compared to the $15 \%$ [7] saving that is achieved through the traditional control of the luminous flux (LC). In this study we did not consider weather conditions which will be the object of future work.

$\mathrm{SL}$ is therefore the optimal solution, combining energy saving with a short payback time.

\section{State of the art}

In order to obtain the maximum possible energy saving and to choose the various solutions to compare, we analyzed all existing and innovative technologies, incorporating both light sources and luminous flux management [5]. In particular, the study focused on lighting management and control and consequently on the energy consumed by the PL system. We analyzed different approaches to luminous flux regulation (two-speed ferromagnetic reactors and centralized and dimmable luminous flux regulators), remote monitoring and remote management systems, as well as an innovative approach based on adaptive systems. We analyzed the data collected through the Lumière project (a Network of 400 municipalities, of which about 200 provided information on their PL system) in order to gain an idea of the starting position of the Italian system and to develop assessment methods and performance indicators for the PL of small and medium towns.

This analysis showed that more than $80 \%$ of the installed power is not controlled by any luminous flux management system (stabilizers and regulators) and that more than $40 \%$ of light sources are inefficient [8] mercury vapor (HG) lamps.

As previously mentioned, the current intervention strategies consist of the following choices:

1. Line control. This consists of replacing HG lamps with high pressure sodium (HPS) lamps, and includes the installation of astronomical clocks and line regulators of luminous flux for those switchboards that manage sufficient electrical power to justify the cost of the investment. Line regulation only allows all the lamps fed from the same switchboard to be dimmed in the same way. Because it is the most economical solution, it is the solution offered most frequently to municipalities by companies involved in improving PL efficiency.

2. Remote point-to-point control. In addition to replacing the lamps, power line digital communication features (Power Line Communication, PLC) are installed to remotely control the luminous flux of each individual lamp (a regulator for each lamp and a central unit for each switchboard). This solution enables 'Smart 


\section{$2^{\text {nd }}$ European Energy Conference}

Services' because it allows sensors and other systems to be connected to the electrical network and thus to acquire data through PLC.

3. The smart lighting approach.

\section{The Smart Lighting approach}

The 'Smart Lighting' approach derives from remote P2P control, but it introduces an element typical of 'Smart' applications, the principle of adaptivity and 'Energy on demand'.

This approach is based on having 'smart and multifunctional' lamp poles in the PL network, equipped with various sensors (typically cameras). These sensors, using advanced communication systems (high-speed power line networks or Zigbee), interact with an intelligent control unit. This system is able to create a profile of the activities (people, vehicles, emissions) taking place on streets in real time. This enables adaptive and automatic point-to-point flux adjustment to take place all year round.

In addition to greatly enhancing the energy performance of $\mathrm{P} 2 \mathrm{P}$ regulation, this approach opens up considerable prospects for developing the PL network, because it allows the aggregation of many 'Smart Services' on the PL network, resulting in decreased costs and greatly improved performance. In fact, the independent and adaptive management of lighting is only the first step towards fully exploiting the potential this equipment offers. Some of the most interesting 'Smart Services' are:

- traffic monitoring and info-mobility;

- supporting public transport and parking management;

- surveillance, security and emergency management;

- detecting noise and environmental pollution;

- wireless and information services;

- managing, monitoring and assessment of public buildings;

- charging electric vehicles.

The following comparison uses Smart Lighting, a solution similar to the remote P2P control where cameras are placed on every street (one every 500-1000 m) to measure the flow of vehicles and pedestrians and a control system is installed that adjusts the luminous flux, according to rules [7] related to the flow of vehicles.

The report Developing Intelligent Systems for the Management of 'Smart Street' [7] shows the full methodology and the estimated energy savings associated with the use of this technology. It shows that the following assumptions can be made: line control reduces power by $20 \%$ for $45 \%$ of the operating hours, remote management by $33 \%$ for $45 \%$ of the hours, and Adaptive Lighting by $45 \%$ for $93 \%$ of the hours. Moreover, stabilizing the voltage reduces the power by $10 \%$ and astronomical clocks reduce the number of operating hours from 4,198 to 4,000 per year.

\section{Technical and economic analysis of a real case of the intervention strategies}

To compare the three solutions mentioned, the Municipality of Castelnuovo Magra (Province of La Spezia) was selected, where an energy audit of the PL system was carried out that provides the basic data necessary for a technical and economic comparison. The Municipality has these characteristics:

-Population: 8251. 
- Area: $15 \mathrm{~km}^{2}$.

-Lamps: 868, of which: HG lamps: 615 (71\%), HPS lamps: 141 (16\%).

- Total power: $135 \mathrm{~kW}$.

-Energy consumption: $562 \mathrm{MWh} / \mathrm{y}$.

- Power cost: $€ 4,826 /$ year. (A)

-Energy cost: €78,664 /year. (B)

- Total energy cost: $€ 83,490 /$ year $(\mathrm{A}+\mathrm{B})(\mathrm{C})$

- Maintenance cost: $€ 25,225$ /year. (D)

- Total cost: $€ 108,715$ /year. (C+D)

Table 1 shows in detail the technical and economic parameters for the current situation and for the three solutions presented.

Table 1. Comparison of the various solutions.

\begin{tabular}{|l|l|l|l|l|l|}
\hline & Current situation & LC & P2P & SL & \\
\hline Total power & 134.6 & 119.7 & 119.7 & 119.7 & $\mathrm{~kW}$ \\
\hline Energy consumption & 562 & 408 & 386 & 288 & $\mathrm{MWh} / \mathrm{y}$ \\
\hline TOE consumption & 105 & 76 & 72 & 54 & $\mathrm{TOE} / \mathrm{y}$ \\
\hline \multirow{2}{*}{ Energy saving } & - & 155 & 176 & 274 & $\mathrm{MWh} / \mathrm{y}$ \\
\cline { 2 - 6 } & - & 27 & 31 & 49 & $\%$ \\
\hline Emission saving (CO2) & - & 91 & 104 & 162 & tCO2/y \\
\hline Total energy cost (C) & 83,490 & 61,333 & 58,355 & 44,608 & $€ / \mathrm{y}$ \\
\hline Maintenance cost (D) & 25,225 & 16,147 & 14,137 & 12,450 & $€ / \mathrm{y}$ \\
\hline Total cost (C+D) & 108,715 & 77,480 & 72,492 & 57,058 & $€ / \mathrm{y}$ \\
\hline \multirow{2}{*}{ Total cost saving } & - & 31,235 & 36,223 & 51,657 & $€ / \mathrm{y}$ \\
\cline { 2 - 6 } & - & 29 & 33 & 48 & $\%$ \\
\hline Cost of investment & - & 148,365 & 230,942 & 307,742 & $€$ \\
\hline Cash flows & - & 31,235 & 36,223 & 51,657 & $€ / \mathrm{y}$ \\
\hline NPV & - & 198,916 & 171,807 & 266,608 & $€$ \\
\hline PBT & - & 5.37 & 7.51 & 6.94 & $\mathrm{y}$ \\
\hline Security level & 3 & 1 & 2 & 3 & \\
\hline
\end{tabular}

The reduction in energy consumption varies from $27 \%$ (LC) to $49 \%$ (SL) and the reduction in emissions ranges from 91 to 162 tons of carbon dioxide per year.

The decrease in fixed costs is guaranteed by the lower power usage of the lamps installed (through the use of HPS), and the variable costs are guaranteed to decrease due to the reduction in the number of operating hours per year and, when planned, the positive effects of stabilizing and adjusting the luminous flux. The decrease in maintenance costs is derived from the longer duration of HPS, which reduces the number of replacements per year, from the reduction in equivalent operating hours, and from the reduction in the costs of finding faults in the remote controlled lamps. In addition, the reduction in the annual number of lamp replacements means there is less material in need of disposal, and in particular means that there are no lamps containing highly dangerous mercury to dispose of.

Thanks to energy efficiency, PL total costs are reduced from the current 108,715 euros to 77,480 euros with LC, to 72,492 euros with remote P2P control and to 57,058 euros with SL.

\section{Benchmarking}

The graph in Fig. 1 shows the trend of the net present value (NPV) through time. This shows, obviously in addition to the NPV at the end of the economic initiative, the payback time (PBT) and the cost of investment. 


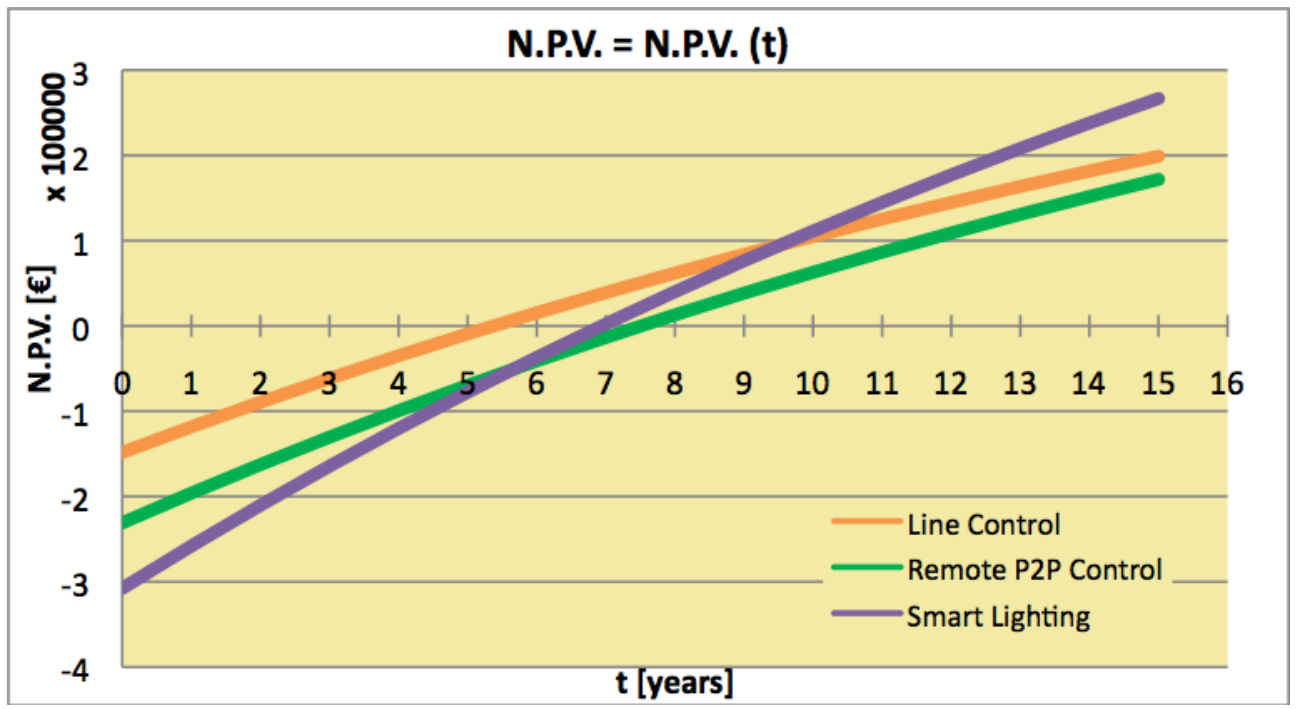

Fig. 1. NPV for the three solutions considered.

The line control has an NPV of around 200,000 euros and a PBT of almost 5.5 years, whilst the remote P2P control is less effective from an economic point of view, with an NPV of 170,000 euros and a PBT of almost 7.5 years. The Smart (Adaptive) Lighting is very competitive, with an NPV of 270,000 euros and a PBT of 7 years. The investment costs range from 150,000 euros for the line control, to 230,000 euros for the remote P2P control and to 300,000 euros for the Adaptive control.

Fig. 2 shows the energy savings (\%), the PBT and the security level (represented by the size of the bubbles) that the various solutions can provide. Control line, which from an economic point of view would seem to be better than remote P2P control, is shown to have a lower energy saving and security level than the other two solutions (P2P and SL).

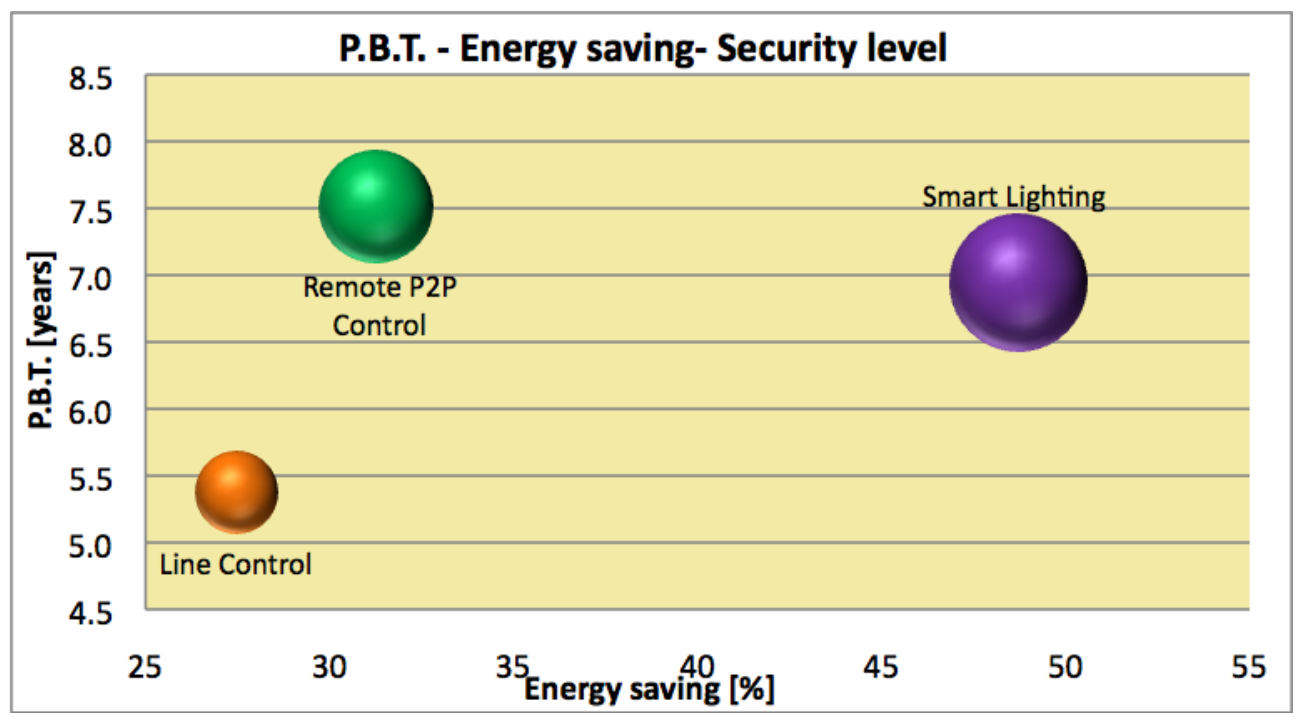

Fig. 2. Energy savings and PBT of the three cases considered. 
From this analysis we can see that the final, 'Smart' solution can be regarded as a logical, natural and competitive evolution of remote P2P control rather than an alternative to it. Adaptive Lighting is not only the better overall solution from an economic standpoint, but also in terms of energy saving and the quality of service offered.

With this solution, the more traffic there is at times with traffic regulation compared to other traffic flow values at the same time, the higher the savings, since it is precisely at this peak that the traditional strategies set their luminous flux reduction value.

Moreover, thanks to the cameras, in addition to lighting control, Smart Lighting can easily be integrated into another two Smart Services like traffic monitoring and video surveillance, and may incorporate other services of great economic, social and environmental interest with very sustainable investments.

\section{Extrapolation of the study into Smart Lighting}

Finally, we can estimate the impact on Italy of a public lighting systems upgrade in all the municipalities that have joined the Lumière Network.

For this purpose, a calculation model was developed to simulate the extrapolation of the effects of the application of Adaptive Lighting technologies based on the study carried out in Castelnuovo (see Table 2). For the municipalities for which we have sufficient data (68 municipalities), the total power, including wastage, is $14.3 \mathrm{MW} .40 .8 \%$ of the light fixtures are equipped with HG lamps, and PL systems are switched on for an average of 4,200 hours per year. The calculation model assumes that the average HPS lamps which replace the HG lamps use $25 \%$ less nominal power for the same luminous flux emitted and that is possible to control about $75 \%$ of the total power.

Table 2. Extrapolation of the study to larger spatial scales.

\begin{tabular}{|c|c|c|c|c|}
\hline & Castelnuovo & Lumière Network & Italy & \\
\hline \multicolumn{5}{|l|}{ Ex-ante situation } \\
\hline Current total power & 134 & 14,339 & $1,452,381$ & $\mathrm{~kW}$ \\
\hline Current power cost $(\mathrm{E})$ & 4,826 & 516,189 & $52,285,714$ & $€ / y$ \\
\hline Current energy cost $(\mathrm{F})$ & 561,886 & $60,222,055$ & $6,100,000,000$ & $\mathrm{kWh} / \mathrm{y}$ \\
\hline Current total energy cost $(\mathrm{E}+\mathrm{F})$ & 78,664 & $8,431,088$ & $854,000,000$ & $€ / y$ \\
\hline \multicolumn{5}{|l|}{ Ex-post situation } \\
\hline Final total power & 119 & 12,877 & $1,304,336$ & $\mathrm{~kW}$ \\
\hline Final power cost $(\mathrm{G})$ & 4,289 & 463,573 & $46,956,100$ & $€ / y$ \\
\hline Final energy cost $(\mathrm{H})$ & 287,990 & $31,126,881$ & $3,152,897,610$ & $\mathrm{kWh} / \mathrm{y}$ \\
\hline Final total energy cost $(\mathrm{G}+\mathrm{H})$ & 40,319 & $4,357,763$ & $441,405,665$ & $€ / y$ \\
\hline \multicolumn{5}{|l|}{ Savings } \\
\hline Power saving & $-15(-11)$ & $-1,462(-10)$ & $-148,045(-10)$ & $\mathrm{kW}(\%)$ \\
\hline Power cost saving & -537 & $-52,616$ & $-5,329,614$ & $€ / y$ \\
\hline Energy saving & -274 & $-29,095$ & $-2,947,102$ & $\mathrm{MWh} / \mathrm{y}$ \\
\hline Energy saving (\%) & -49 & -48 & -48 & $\%$ \\
\hline Energy cost saving & $-38,345$ & $-4,073,324$ & $-412,594,335$ & $€ / y$ \\
\hline
\end{tabular}

Although the initial assumptions are simplifications, we obtained very clear results (see Table 2). With Adaptive Lighting, energy savings can reach $48 \%$, with a reduction of 29 GWh per year in electricity consumption. This value represents almost $0.1 \%$ of the entire Italian demand of about $310 \mathrm{TWh}$ per year [1]. This would mean a reduction in annual carbon dioxide emissions of 17,193 tons, with consequent benefits for the whole community.

In economic terms, there are considerable annual savings; the upgrading would save the municipalities $4,000,000$ euros annually for the energy component of the electric bill alone. 


\section{$2^{\text {nd }}$ European Energy Conference}

With this calculation model we estimated, as a purely qualitative indication, the effects of upgrading all PL systems in Italy. This projection is based on some data and assumptions originating from data collected as part of the Lumière project.

Italian electricity consumption for PL is about 6.1 TWh per year [1], corresponding to a charge of about 854 million euros; assuming the current number of operating hours per year $(4,200)$ of the municipalities in the Network is the same nationwide, this signifies an installed power of $1,452 \mathrm{MW}$.

If it is assumed that the percentage of HG lamps is $41 \%$ and that sodium lamps require $25 \%$ less power, the installed power, thanks to such a substitution, decreases by $148 \mathrm{MW}(10 \%)$. Given remote control of $75 \%$ of installed power with an adaptive system, energy and economic savings would be very significant. Energy consumption would decrease by 2.9 TWh per year (48\%), carbon dioxide emissions would be reduced by 1,741,000 tons per year, and expenditure would fall by almost 413 million euros. To understand the magnitude of energy savings one can compare it with the national demand $(310 \mathrm{TWh} / \mathrm{y})$ - it is equivalent to almost $1 \%$ of this value.

To confirm this result it has been recalculated in two ways, by considering the very reliable results obtained for the Network and extending them to Italy through the relationship between people and area. The average results do not deviate too much from those reported above and summarized in Table 2 .

\section{Conclusions}

In conclusion, we can say that Smart Lighting, which uses information and communication technologies and is based on the transmission of digital data, would have very positive consequences for Italy and for all the municipalities, from an energy, environmental and economic point of view (see Table 3).

Table 3. Summary table of the extrapolation of the study.
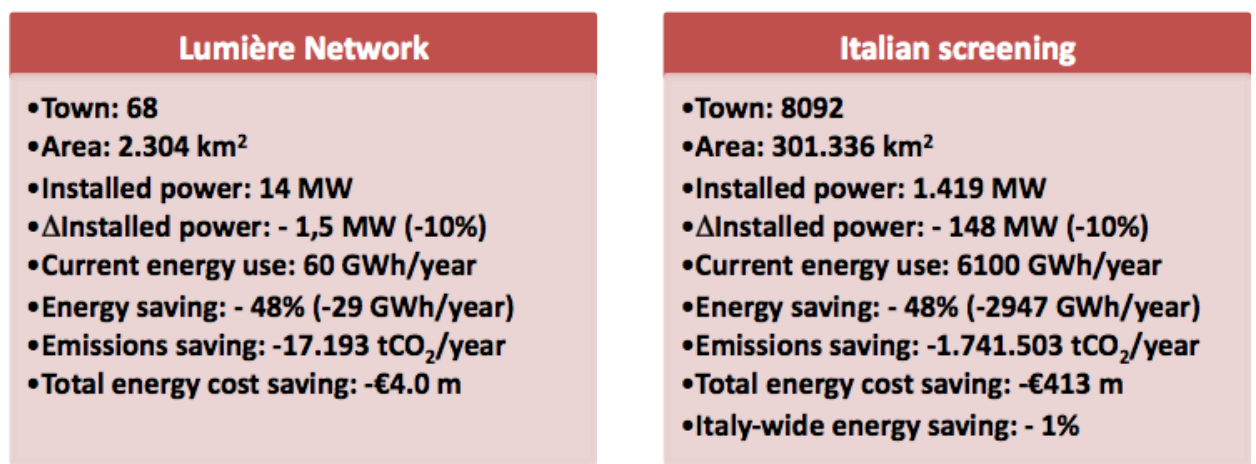

To try to reduce electricity consumption we focused on public lighting. The overview of the current status in terms of technology and instrumentation revealed the need to develop a means of promoting technological innovation and consequently energy efficiency. Indeed, the payback time is inversely proportional to the mass deployment of instrumentation equipment on PL. Additionally, the use of PLC allows information and data processing to be managed centrally with no additional cabling costs, and provides a very high level of computation which is extremely difficult to achieve with local data processing.

The aim of this work was to assess the efficacy and competitiveness of innovative technology for PL that enables Smart Services to be integrated into the electrical network, 
by creating tools and methodologies for technical and economic assessments and piloting the operation strategies on a real case.

We presented technologies for luminous flux management, including systems for turning lamps on and off, for stabilizing and regulating the voltage and for luminous flux remote control.

In the future these technologies will be integrated with other sensors, such as for air quality monitoring, and a system may be developed that controls a sub-network (Smart Street or Smart Square) and then a set of subnets (urban network).

The Adaptive control system goes beyond the concept of the simple hourly adjustment of the luminous flux, adapting the energy delivered to the effective demand at any one moment in order to use energy only when necessary and in the amount required (Energy on Demand). It has been demonstrated in a real case that the Adaptive control strategy can produce an average energy saving of over $40 \%$ compared to the $15 \%$ saving that is achieved with the traditional non-adaptive control of the luminous flux (either with the line control or with remote $\mathrm{P} 2 \mathrm{P}$ control). The control occurs through time adjustment based on a predictive model of vehicle flows.

The application of 'Smart Lighting' would substantially increase Italy's energy efficiency and would provide significant reductions in costs. In addition, as a beneficial side effect, it could make a considerable contribution to achieving the objectives of European and international directives, such as those imposed by the Kyoto Protocol, which provide for the drastic reduction of greenhouse gas emissions. SL allows higher savings in monetary terms, not only for Italy but also for the municipalities, and would create additional technologies accompanied by a significant development not only in lighting, but also in transport, building management, security and in the quality of services offered to citizens and tourists in general.

In conclusion, the introduction of an autonomous and adaptive control system (SL), balanced against a modest initial cost, may lead to a significant increase in energy savings for the entire urban district. In few words Smart Lighting is the access to Smart City.

\section{References}

1. Terna, "Previsioni della domanda elettrica in Italia e del fabbisogno di potenza necessario, anni 2007 - 2017”, Documento Tecnico, 30 Settembre (2007)

2. Annunziato M., Le Smart Cities e le sinergie europee del consorzio European Energy Research Alliance, QualEnergia, giugno-luglio 2011, anno IX, n.3 (2011)

3. Art Valley Association, Le città intelligenti. Scrivendo un manuale di citymatica per amministratori lungimiranti e cittadini esigenti, Art Valley Association (2010)

4. Gozo N. et al, Percorso e soluzioni economico-finanziarie per la realizzazione di interventi di riqualificazione dell'impianto di illuminazione pubblica, Linee Guida, Accordo di Programma ENEA-MSE, (2011)

5. Bucci F., Analisi tecnico-economica di un modello di illuminazione pubblica innovativa basato su tecnologie innovative abilitanti alla SMART CITY, Tesi di laurea in Ingegneria Meccanica, Università degli Studi di Roma Tre, (2011)

6. Cecchini L., Il telecontrollo nell'illuminazione stradale, in "Luce \& Design", (2006)

7. Annunziato M. et al, Sviluppo sistemi intelligenti per la gestione della "Smart Street", rapporto tecnico ENEA-MSE, Ricerca di Sistema Elettrico PAR 2008-2009, (2011)

8. Cammarata G., Illuminotecnica, Dipartimento di Ingegneria Industriale e Meccanica, Università di Catania, (2003) 